\title{
ON THE ORDERS OF CONJUGACY CLASSES IN GROUP ALGEBRAS OF $p$-GROUPS
}

\author{
A. BOVDI, L. G. KOVÁCS and S. MIHOVSKI
}

(Received 19 February 2003)

Communicated by E. O’Brien

\begin{abstract}
Let $p$ be a prime, $\mathbb{F}$ a field of $p^{n}$ elements, and $G$ a finite $p$-group. It is shown here that if $G$ has a quotient whose commutator subgroup is of order $p$ and whose centre has index $p^{k}$, then the group of normalized units in the group algebra $\mathbb{F} G$ has a conjugacy class of $p^{n k}$ elements. This was first proved by $\mathrm{A}$. Bovdi and C. Polcino Milies for the case $k=2$; their argument is now generalized and simplified. It remains an intriguing question whether the cardinality of the smallest noncentral conjugacy class can always be recognized from this test.
\end{abstract}

2000 Mathematics subject classification: primary 16S34, 16U60; secondary $20 \mathrm{C} 05$.

Keywords and phrases: group algebra, group of units, conjugacy class, finite $p$-group.

Let $\mathbb{F} G$ be the group algebra of a finite $p$-group $G$ over the field $\mathbb{F}$ with $p^{n}$ elements. As is well known, the radical $R$ of the group algebra $\mathbb{F} G$ is then just the augmentation ideal

$$
A=\left\{\sum_{g \in G} a_{g} g \mid a_{g} \in \mathbb{F}, \sum_{g \in G} a_{g}=0\right\},
$$

and every element of $\mathbb{F} G$ outside $A$ is a unit. The group of normalized units is

$$
V=V(\mathbb{F} G)=\{1+a \mid a \in A\},
$$

and this is a group of order $p^{n(|G|-1)}$. The structure of such groups $V$ is one of the interesting questions in the theory of modular group algebras. What is the connection of the class of these groups with other classes of finite $p$-groups? Explicit calculations

The research of the first author was partly supported by the Hungarian National Fund for Scientific Research grant No. T037202, and that of the second author by Plovdiv University under contract M 25/2002.

(C) 2004 Australian Mathematical Society 1446-7887/04 \$A2.00+0.00 
in $V(\mathbb{F} G)$ are usually extremely difficult, even when $G$ is fairly small, so examples are very hard to come by.

The survey [1] covered many relevant results; more have appeared since then, for example in [3] and [4].

In this paper we continue an investigation, begun by Polcino Milies and the first author in [2], exploring what numbers can occur as cardinalities of conjugacy classes of elements of $V$. Only powers of $p^{n}$ come into consideration, because the cardinality of the conjugacy class of an element $v$ is the index of the centralizer $C_{V}(v)$ while

$$
C_{V}(v)=\left\{1+a \mid a \in C_{A}(v)\right\}
$$

where $C_{A}(v)$ is an $\mathbb{F}$-subspace, so in fact

$$
\left|V: C_{V}(v)\right|=p^{n\left(|G|-1-\operatorname{dim} C_{A}(v)\right)}=p^{n\left(|G|-\operatorname{dim} C_{\mathrm{FG}}(v)\right)} .
$$

To each element $x=\sum x_{g} g$ of the group algebra, $x+\left(1-\sum x_{g}\right)$ is an element of $V$, with the same centralizer as $x$, so our question may be thought of as this: what numbers can occur as codimensions of centralizers of elements in the group algebra $F G$ ?

It was shown in [2] that 1 cannot occur as such a codimension, but 2 always does if $G$ has a nonabelian quotient in which the index of the centre is $p^{2}$. Of course, then 2 is the smallest positive codimension, and the commutator subgroup of the quotient in question has order $p$. Every finite nonabelian $p$-group $G$ has quotients with commutator subgroups of order $p$; let $p^{k}$ be the index of the centre in such a quotient. Our main result is that in this case $\mathbb{F} G$ has an element whose centralizer has codimension $k$. However, we have not been able to decide whether the smallest positive codimension must always arise in this way (that is, by considering all such quotients and choosing one with centre of minimal index).

THEOREM. Let $\mathbb{F}$ be a field of $p^{n}$ elements and $G$ a finite $p$-group. If $G$ has a quotient with commutator subgroup of order $p$ and centre of index $p^{k}$, then the group of normalized units of $\mathbb{F} G$ has a conjugacy class of $p^{\text {nk }}$ elements.

QUESTION. If $k$ is chosen minimal for $G$, is $p^{k n}$ minimal among the cardinalities of the conjugacy classes of noncentral elements?

By the above discussion, the Theorem will follow once we prove the two lemmas below. The first of these is very general.

LEMMA 1. Let $\mathbb{F}$ be any field, $G$ any finite group, $G \rightarrow \bar{G}$ a surjective homomorphism, $r(\in \mathbb{F} G)$ the sum of the elements of the kernel, and $\mathbb{F} G \rightarrow \mathbb{F} \bar{G}, x \mapsto \bar{x}$ the corresponding homomorphism of $\mathbb{F}$-algebras. For each element $x$ of $\mathbb{F} G$, the codimensions of the centralizers of $x r$ and $\bar{x}$ are equal:

$$
|G|-\operatorname{dim} C_{\mathbb{F} G}(x r)=|\bar{G}|-\operatorname{dim} C_{\mathbb{F} \bar{G}}(\bar{x}) .
$$


LEMMA 2. Let $\mathbb{F}$ be any field of characteristic $p$, and $G$ a finite $p$-group with commutator subgroup $\langle c\rangle$ of order $p$ and centre $Z$ of index $p^{k}$. Further, let $T$ be a transversal of $\langle c\rangle$ in $G$, and set $s=\sum_{t \in T} t$. Then the centralizer of the element $s(c-1)^{p-2}$ in $\mathbb{F} G$ has codimension $k$. (When $p=2$, interpret $(c-1)^{p-2}$ as 1.)

Under the assumptions of the Theorem, one could now write down explicitly a normalized unit with the required number of conjugates; this is left to the reader.

We remark here that $k$ is always even. For, if the commutator subgroup of a finite $p$-group is of order $p$, then it is contained in the centre, and the central factor group is elementary abelian. Moreover, $(g Z, h Z) \mapsto g^{-1} h^{-1} g h$ yields a nondegenerate symplectic form on $G / Z$ viewed as a vector space over the field of $p$ elements, so the dimension $k$ of this space is always even.

The proofs of the lemmas simplify and generalize some of the arguments from [2], but there remain results of interest in that paper that have not been mentioned here.

PROOF OF LemMa 1. Step 1. The sum $r$ is central in $\mathbb{F} G$, because when $r$ is conjugated by an element of $G$, only the order of the summands changes.

Step 2. For $x \in \mathbb{F} G, x r=0$ is equivalent to $\bar{x}=0$. To see this, let $K$ be the kernel and $T$ be a transversal to the kernel. Each element $x$ of $\mathbb{F} G$ is of the form $x=\sum_{t \in T} \sum_{h \in K} x_{t h} t h$, and $h r=r$ for every $h$ in $K$ (because when the sum $r=\sum_{k \in K} k$ is multiplied by $h$, only the order of the summands changes). Thus the coefficient of $t h$ in $x r$ is $\sum_{k \in K} x_{t k}$, independent of $h$, and it is also the coefficient of $\bar{t}$ in $\bar{x}$.

Step 3. For $x, y \in \mathbb{F} G,(x r) y=y(x r)$ if and only if $\bar{x} \bar{y}=\bar{y} \bar{x}$. Indeed, $(x r) y-$ $y(x r)$ is $(x y-y x) r$ by Step 1 , so by Step 2 it vanishes if and only if $\overline{x y-y x}$ does.

Step 4. Step 3 means that $C_{\mathbb{F} G}(x r)$ is the complete inverse image of $C_{\mathrm{F}} \bar{G}(\bar{x})$ under the homomorphism $\mathbb{F} G \rightarrow \mathbb{F} \bar{G}$. Since this homomorphism is surjective, it follows that the codimensions of the two centralizers are equal.

PROOF OF LEMMA 2. Recall that assumptions and notations are not the same as in the previous proof. In particular, now $T$ is a transversal to $\langle c\rangle$, while $s=\sum_{t \in T} t$ and $c \in Z$. Elementary calculations show that $c^{i}(c-1)^{p-1}=(c-1)^{p-1}$ for every element $c^{i}$ of the commutator subgroup $\langle c\rangle$. For short, write $s(c-1)^{p-2}=y$.

For $g \in G$ and $t \in T$, we may write $g^{-1} t g=t c^{i_{1}}$ with $0 \leq i_{t}<p$, and then

$$
\begin{aligned}
g^{-1} y g=\sum_{i \in T} t c^{i_{i}}(c-1)^{p-2} & =y+\sum_{i \in T} t\left(c^{i_{t}-1}+c^{i_{1}-2}+\cdots+1\right)(c-1)^{p-1} \\
& =y+\sum_{t \in T} i_{t} t(c-1)^{p-1} .
\end{aligned}
$$

If $g^{-1} y g=y$, then $i_{t}=0$ for all $t \in T$. This proves that $C_{G}(y)=Z$. 
The ideal $\mathbb{F} G(c-1)^{p-1}$ lies in the centre of $\mathbb{F} G$. For, this ideal is spanned by the elements $g(c-1)^{p-1}$ with $g \in G$; when such an element is conjugated by some element of $G$, it is multiplied by an element of the commutator subgroup, and (as we have already noted) every such factor is absorbed by $(c-1)^{p-1}$.

Modulo this ideal, $h y \equiv y$ whenever $h \in G$. To see this, recall that the coset of $y$ modulo $\mathbb{F} G(c-1)^{p-1}$ is the sum of the cosets of the $t(c-1)^{p-2}$ as $t$ ranges through $T$. When this sum is multiplied by $h$, only the order of the summands changes, because if $h t=t^{\prime} c^{i}$ for some $t^{\prime}$ in $T$ and $c^{i} \in\langle c\rangle$, then

$$
\begin{aligned}
h t(c-1)^{p-2}=t^{\prime} c^{i}(c-1)^{p-2} & =t^{\prime}(c-1)^{p-2}+t^{\prime}\left(c^{i}-1\right)(c-1)^{p-2} \\
& \equiv t^{\prime}(c-1)^{p-2} \quad\left(\bmod \mathbb{F} G(c-1)^{p-1}\right) .
\end{aligned}
$$

As a consequence we have that every product $(h-1) y$ is central in $\mathbb{F} G$, and by a similar argument so is every $y(g-1)$ with $g \in G$. It follows that

$$
(g-1)(h-1) y=(h-1) y(g-1)=y(g-1)(h-1),
$$

whence

$$
(g h) y-y(g h)=(g y-y g)+(h y-y h) .
$$

This shows that the map $g \mapsto g y-y g$ is a homomorphism from the multiplicative group $G$ to the additive group of $\mathbb{F}_{p} G$, where $\mathbb{F}_{p}$ is the prime subfield of $\mathbb{F}$. We have seen that the kernel $C_{G}(y)$ of this homomorphism is precisely the centre $Z$.

The map $x \mapsto x y-y x$ is an endomorphism of the $\mathbb{F}$-space $\mathbb{F} G$, with kernel $C_{\mathrm{F} G}(y)$ and image the $\mathbb{F}$-span of the $g y-y g$ with $g \in G$. Thus the codimension of $C_{\mathrm{F} G}(y)$ is the dimension of this $\mathbb{F}$-span. The $g y-y g$ all lie in $\mathbb{F}_{p} G$, and there they form an additive subgroup isomorphic to $G / Z$, that is, an $\mathbb{F}_{p}$-subspace of dimension $k$. Since $\mathbb{F} G$ arises from $\mathbb{F}_{p} G$ simply by extension of scalars, it follows that the $\mathbb{F}$-span of the $g y-y g$ is also of dimension $k$. This completes the proof.

\section{References}

[1] A. Bovdi, 'The group of units of group algebras of characteristic p', Publ. Math. Debrecen 52 (1998), 193-244.

[2] A. Bovdi and C. Polcino Milies, 'Conjugacy classes of the group of units in group algebras of finite p-groups', An. Ştiint. Univ. Ovidius Constanfa Ser. Mat. 8 (2000), 1-12.

[3] —-, 'Normal subgroups of the group of units in group rings of torsion groups', Publ. Math. Debrecen 59 (2001), 235-242.

[4] V. Bovdi and M. Dokuchaev, 'Group algebras whose involutory units commute', Algebra Colloq. 9 (2002), 49-64. 
University of Debrecen

Australian National University

4010 Debrecen

Canberra ACT 0200

Hungary

Australia

e-mail: bodibela@math.klte.hu

e-mail: kovacs@maths.anu.edu.au

University of Plovdiv

4000 Plovdiv

Bulgaria

e-mail: mihovski@uni-plovdiv.bg 
J. Aust. Math. Soc. 77 (2004) 